\title{
Foamability and Foam Stability of Several Surfactants Solutions: The Role of Screening and Flooding
}

\section{Azmi Belhaij and Osama Al-Mahdy*}

King Saud University, Abdulrahman AlQuraishi, King Abdulaziz City for Science and Technology, Osama Al-Mahdy, King Saud University

\begin{abstract}
Foamability and foam stability are of main concerns in foam displacement for enhanced oil recovery. This work presents the output of systematic laboratory screening of foamability and foam Stability of several surfactants. The surfactants examined were Brij 700, Triton X-100, Triton X-405, Zonyl FSO, Hitenol H-10, Hitenol H-20, Noigen N-10 and Noigen N-20. Foam was generated by sparging Carbon Dioxide gas at a fixed flow rate through surfactants solutions and R5 parameter as suggested by Lunkenheimera and Malysa (2003) were used for foam stability testing. The results indicate the foamability of all surfactants except for Triton X-405. Zonyl FSO and Hitenol H-10 were superior in term of foam stability with more stability as surfactants concentration increases. Equivalent optimum foam volumes were obtained for both surfactants but at higher concentrations of Hitenol H-10.

Foam stability and oil displacement efficiency were tested with different concentrations of Zonyl FSO and Hitenol $\mathrm{H}-10$ solutions. The presence of oil at the volume fraction implemented, affect the stability of the foam columns. The effect depends on the surfactant-type and surfactants concentrations where stability decreases at low Zonyl FSO concentration range and at all concentrations range tested of Hitenol $\mathrm{H}-10$. In case of Zonyl FSO observations indicate that oil stayed in the lamellas skeleton and plateau boarders with no drain out. To the contrary, Hitenol $\mathrm{H}-10$ was able to lift good portion of the oil column but oil was drained out of the foam structure within a short period of time.

Flooding tests on Berea cores proved the ability of Zonyl FSO and Hitenol $\mathrm{H}-10$ in controlling gas mobility and improving the displacement efficiency. Hitenol $\mathrm{H}-10$ was more efficient as indicated by the incremental oil recovery obtained and the higher pressure drop encountered. Hitenol $\mathrm{H}-10$ Foam injection on tertiary gas flooded reservoirs improves residual oil recovery indicating the potential of the process even at late stages of gas injection.
\end{abstract}

Keywords: Triton X-100; Enhanced oil recovery; Enhanced oil recovery

\section{Introduction}

The declining trend of new discoveries coupled with the high demand for energy, directed the industry to the importance of enhanced oil recovery (EOR) processes in low recovery efficiency reservoirs. Gas flooding is a common method implemented for improved oil recovery. Despite the favorable characteristics of dense carbon dioxide in displacing oil from reservoir rocks, viscous fingering, gravity override and reservoir heterogeneity are the main disadvantages of $\mathrm{CO}_{2}$ floods. Foam injection as an EOR method was first introduced in 1958 by Bond and Holbrook [1]. Later, Fried [2] proved that foam can act as gas blocking agent. Foam displays favorable flow characteristics to EOR process including favorable mobility, selective blocking of thief zones and flow diversion to lower permeability regions [3-6].

Foams are usually formed in systematic hexagonal texture as a result of gas dispersion through a continuous surfactant solution [7]. It is thermodynamically unstable and they are stabilized by surfactants to prevent bubble coalescence. Foams are generally described in terms of their foamability defined as the capacity of the surfactants to form foam irrespective of the special foam properties, and foam stability describing the variations of foam height or volume with time, immediately after foam generation [8]. Foamability and foam stability are interrelated and the more stable the foam films the greater is the solution's foamability.

Foam generation generally increases with increasing surfactant concentration up to the critical micelle concentration (CMC) above which surfactant concentration has little effect [9]. Marsden and Khan [10] found that foam apparent viscosity increases with increasing surfactant concentration. Foam stability is dependent on electric double-layer repulsion [11,7], gravity drainage, capillary suction, surface elasticity, dispersion force attraction, steric repulsion and surface and bulk viscosity [7]. An increased surface and bulk viscosity do not contribute directly to film stabilization. They rather act as resistances to the film thinning and rupturing processes.

Foam stability in presence of oil is related to foam-oil interaction. Literatures indicated the role of oil presence on foam stability [7, 12 15]. Wasan et al. [16] indicated that foam stability in presence of oil is related to pseudo emulsion film between oil drops and gas. Vikingstad et al. [17] performed static foam tests and observed that foam stability in presence of oil is related to surfactant ability to solubilize oil molecules. Oils characterized with higher molecular weight can stabilize the generated foam compared to lower molecular weight oils.

Three major mechanisms have been considered for the antifoaming property of the dispersed oils. These are aqueous film thinning rate during oil entry, oil spreading on the water surface, and thin water film bridging $[18,19]$. Simjoo et al. [20] suggested that foam decay in presence of oil starts as small and rapid decay dominated by gravity drainage, followed by a stabilized foam volume, and then a second

*Corresponding author: Osama Al-Mahdy, King Saud University, Abdulrahman AlQuraishi, King Abdulaziz City for Science and Technology, Tel: 00966548156216; E-mail: omahdy@ksu.edu.sa

Received April 18, 2015; Accepted May 06, 2015; Published May 13, 2015

Citation: Belhaij A, Mahdy OA (2015) Foamability and Foam Stability of Several Surfactants Solutions: The Role of Screening and Flooding. J Pet Environ Biotechnol 6: 227. doi:10.4172/2157-7463.1000227

Copyright: ( 2015 Belhaij A, et al. This is an open-access article distributed under the terms of the Creative Commons Attribution License, which permits unrestricted use, distribution, and reproduction in any medium, provided the original author and source are credited. 
continuous decay due to bubble coalescence over a relatively long time. The oil foam destabilization is more pronounced for oils with a smaller molecular weight. Farajzadeh et al. [6] showed that $\mathrm{CO}_{2}$ injection below the gas minimum miscibility pressure hardly foams in the oil zone of the porous medium.

This work aimed to screen several surfactants investigating their foamability and foam stability in absence and presence of Saudi light crude oil. The efficiency of the most effective surfactants in controlling the gas mobility is tested in porous medium.

\section{Materials}

Several surfactants were screened. These surfactants are nonionic (Brij 700, Triton X-100, Triton X-405, and Zonyl FSO, Noigen N-10 and Noigen $\mathrm{N}-20$ ), and anionic (Hitenol H-10, Hitenol H-20). Surfactant solutions of different concentrations were prepared by diluting them in brines of $4 \%$ salinity. Table 1 lists the compositions of brine solutions. Oleic phase was light Saudi crude (31.5 API, $9 \mathrm{cp}$ ) while high purity Carbon dioxide gas was used as gaseous phase. Porous medium were homogeneous Berea sandstone core samples $10 \mathrm{~cm}$ long with $3.8 \mathrm{~cm}$ diameter.

\section{Experimental Procedure}

Foam stability of surfactant solutions were examined using the R5 method proposed by Lunkenheimera and Malysa [21]. The setup used (Figure 1) consists of a glass column $100 \mathrm{~cm}$ long with $1.6 \mathrm{~cm}$ inner diameter equipped with glass frit placed at the base of the column. A syringe was used for gas supply into the solution in order to generate the required foam. The experiments are conducted by slowly pouring surfactant solution $(12.5 \mathrm{~mL})$ into the glass column and manual introduction of Carbon dioxide gas $(50 \mathrm{~mL})$ into solution within a period of 20 seconds. After gas introduction, the stopcock connecting the column with the syringe is immediately closed, and the initial foam and solution heights are measured. Foam height and solution level are then measured at five minutes time in order to determine the R5 parameter defined as the ratio of foam height at five minutes time to initial foam height. The test was conducted for each surfactant at different concentrations in absence of oil. The most effective surfactants were determined and their foamability and stability were further investigated in presence of crude oil.

A series of flooding runs were conducted using Core Flooding System - CFS 200 (Figure 2) in order to test the foam efficiency in improving oil recovery. It consists of core holder capable of housing core samples up to $30.5 \mathrm{~cm}$. The core holder is tapped with equally spaced high precession pressure transducers for pressure measurements across the core length. Fluids (brine, oil and surfactant solutions) are injected using ISCO positive displacement pump while gas $\left(\mathrm{CO}_{2}\right)$ is flown through flow controller/meter. Confining pressure was applied using hydraulic pump while pore pressure is implemented at the core outlet using dome type back pressure regulator. All experiments were conducted at room temperature and confining and pore pressures of 1000 psi and 200 psi respectively.

\begin{tabular}{|c|c|}
\hline Component & Weight (\%) \\
\hline $\mathrm{NaCl}$ & 3.06 \\
\hline $\mathrm{KCl}$ & 0.029 \\
\hline $\mathrm{CaCl}_{2}$ & 0.54 \\
\hline $\mathrm{MgCl}_{2} \cdot 6 \mathrm{H}_{2} \mathrm{O}$ & 0.26 \\
\hline $\mathrm{Na}_{2} \mathrm{SO}_{4}$ & 0.3 \\
\hline $\mathrm{H}_{2} \mathrm{O}$ & 95.81 \\
\hline
\end{tabular}

Table 1: Composition of $4 \%$ brine.
Flooding experiment starts with core sample inserted in the core holder. Confining and pore pressure are applied and the sample is evacuated and pressure saturated for porosity measurement. The sample is flown with brine solution at different rates and pressure drop across the core samples are reordered and Darcy law is used to determine the core permeability. Sample is then injected with oil to irreducible water saturation and left overnight. The sample is then secondary flooded with brine until oil production ceases. Tertiary flooding is started by injecting a slug of 0.2 pore volumes of surfactant solutions followed by continuous injection of $\mathrm{CO}_{2}$ gas. Recovery and pressure drop is monitored and recorded to test the generated foam efficiency in controlling gas mobility and improving recovery. To test the potential of foam injection in tertiary gas flooded reservoir, one more run was conducted subjecting core sample to secondary water flooding and tertiary gas injection. The residual oil was targeted by slug of 0.2 pore volume of most efficient foaming agent followed by continuous gas injection.

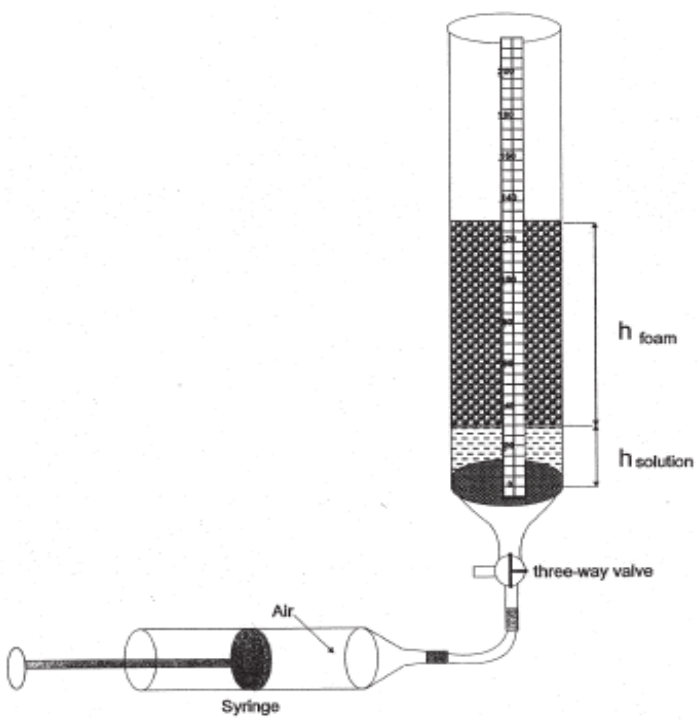

Figure 1: Schematic of foamability and foam stability experiment apparatus.

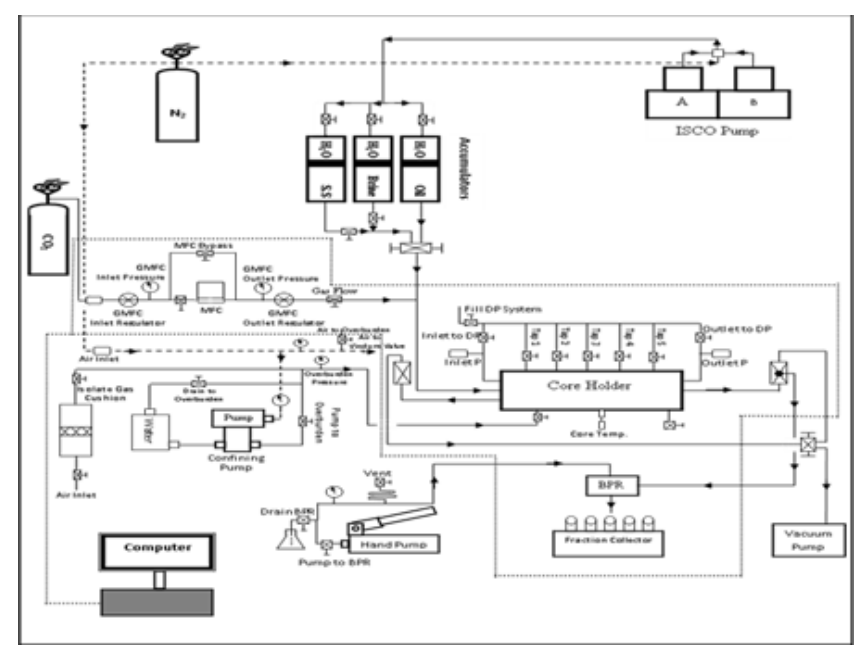

Figure 2: Schematic of flooding unit. 
Citation: Belhaij A, Mahdy OA (2015) Foamability and Foam Stability of Several Surfactants Solutions: The Role of Screening and Flooding. J Pet Environ Biotechnol 6: 227. doi:10.4172/2157-7463.1000227

\section{Results and Discussion}

To investigate the foam stability during the foam formation, each surfactant concentration was introduced with gas volumes of 25 and $50 \mathrm{cc}$ during time period of 20 seconds. This corresponds to average volumetric rates of 4.5 and $9 \mathrm{~L} / \mathrm{hr}$. Figure 3 is an example plot of initial foam height of Hitenol $\mathrm{H}-10$ surfactant solutions as a function of surfactant concentration. The figure shows a two times greater foam height when gas injection was doubled from $4.5 \mathrm{~L} / \mathrm{hr}$ to $9 \mathrm{~L} / \mathrm{hr}$ indicating an initial foam volume equivalent to the dispersed gas and solution volumes lifted by the foam generated. Hence, a stable foam structure was formed with no significant rupture. Figure 4 summarizes the results for all surfactants solution investigated. All can be described with good foamability during foam column formation except for Triton X-405 where foam height was increased by only $50 \%$ when gas rate of injection was doubled.

The half time decay $(\mathrm{t} 1 / 2)$ of foam column is the most common method used to test foam stability. This method can be time consuming, hence R5 method was used for quick evaluation of foam stability. Figure 5 plots the R5 values as a function of surfactants concentrations for all surfactants solutions. Except for the Zonyl FSO and Hitenol H-10, all tested surfactants solutions are characterized as poor stability foamers

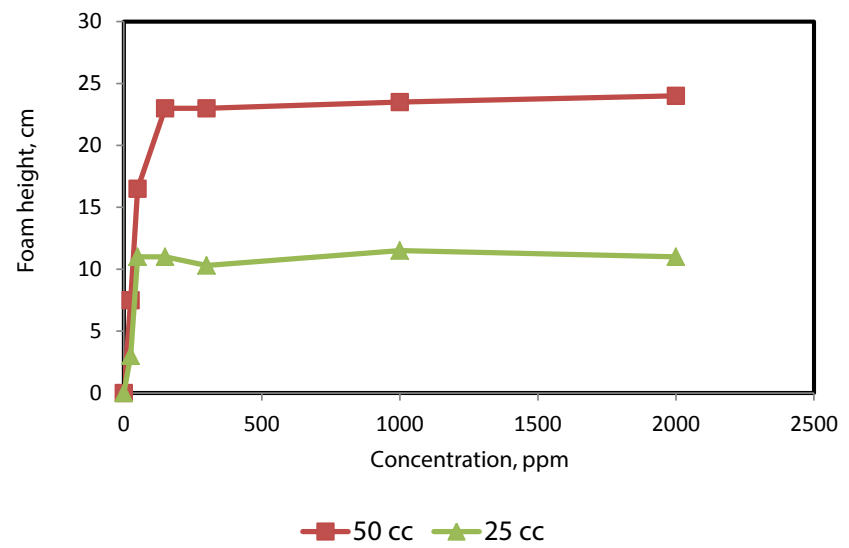

Figure 3: Foam height versus surfactant concentration of $4 \%$ salinity Hitenol $\mathrm{H} 10$ solutions at 25 and $50 \mathrm{cc}$ of gas injection.

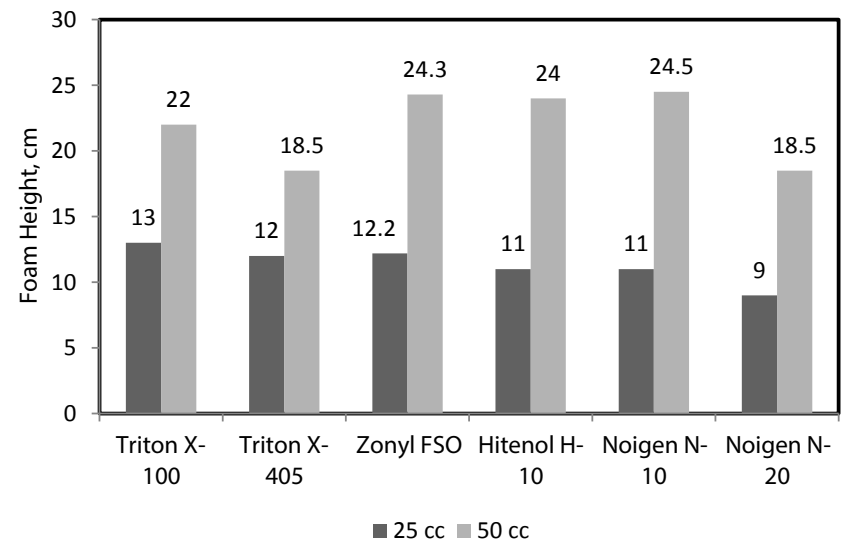

Figure 4: Foam height versus surfactant concentration of $4 \%$ salinity surfactant solutions at 25 and $50 \mathrm{cc}$ of gas injection. with full foams rupture at a very short time frame of less than 5 minutes. Zonyl FSO provided a constant R5 value of $65 \%$ at 200 ppm surfactant concentrations and above. Hitenol H-10 surfactant solutions indicate a low $\mathrm{R} 5$ values below $50 \mathrm{ppm}$ concentrations with a sharp increase in R5 between $50 \mathrm{ppm}$ and $150 \mathrm{ppm}$ and a constant R5 value around $60 \%$ above $150 \mathrm{ppm}$. Accordingly both surfactants are considered metastable as indicated by $\mathrm{R} 5$ values above $50 \%$ with transition from low stability to high stability within a range of surfactant concentrations. Zonyl FSO required a wider range of concentrations to reach stability compared to Hitenol $\mathrm{H}-10$ and that can be related to the different surface activities of the two surfactants.

A major concern when applying foam for mobility control is its stability in the presence of oil. Foam stability of Zonyl FSO and Hitenol $\mathrm{H}-10$ solutions at $4 \%$ salinity were tested in presence of light crude oil. Results obtained were compared with those obtained previously in absence of oil. Figures 6 and 7 compare the foam stability of Zonyl FSO and Hitenol $\mathrm{H}-10$ respectively in presence and absence of crude oil. In presence of oil, Zonyl FSO solutions provide lower foam height at lower concentrations (Figure 6). No effect was seen at concentrations above $100 \mathrm{ppm}$. Observations indicate a good foam height but with some gap in the lower portion of the foam column and low oil lift at concentrations on the range 100 to $300 \mathrm{ppm}$. Above $600 \mathrm{ppm}$ a good

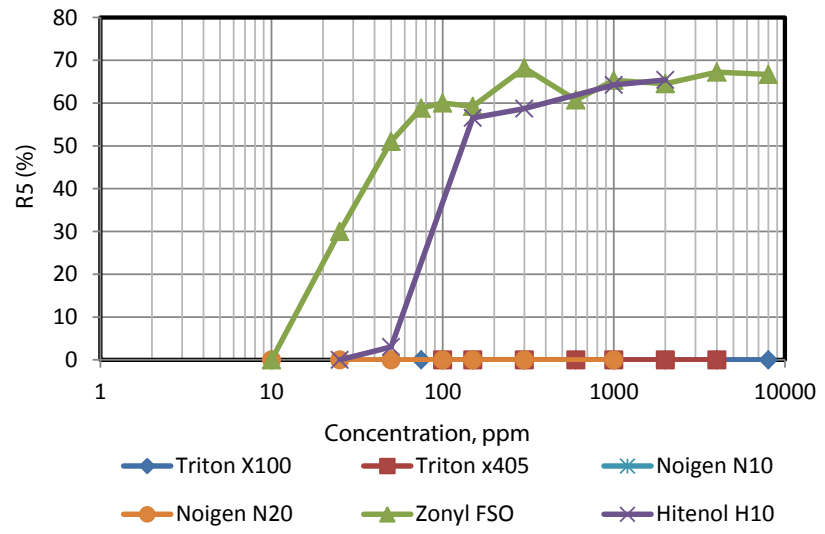

Figure 5: Variation of R5 as a function of surfactant concentration of differen tested surfactants.

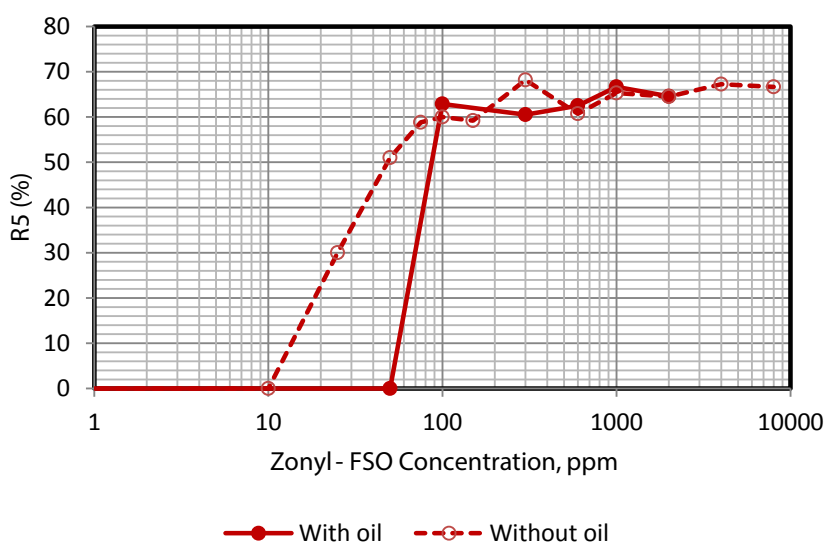

Figure 6: Variation of R5 as a function of surfactant concentration of Zony FSO in presence and absence of oil. 
Citation: Belhaij A, Mahdy OA (2015) Foamability and Foam Stability of Several Surfactants Solutions: The Role of Screening and Flooding. J Pet Environ Biotechnol 6: 227. doi:10.4172/2157-7463.1000227

Page 4 of 6

foam structure was obtained with good stability and efficiency in lifting good portion of the oil at the lamellas skeleton and plateau boarders with no drainage as shown in Figure 8. Figure 7 showed drastic lower foam height for Hitenol $\mathrm{H}-10$ with very week stability in which foam vanishes as gas injection ceases at concentrations around $100 \mathrm{ppm}$. As concentration increases within the range of 150 to $300 \mathrm{ppm}$, good foam height was observed but with week structure in which foam vanishes completely within the first minute. As concentration increases above $1000 \mathrm{ppm}$ we obtained a good foam height with reasonable stability and oil displacing efficiency. Figure 8 presents a snapshot showing oil phase distribution inside the foam structure accumulating at the plateau borders indicating the strength of Hitenol H-10 foam films. However, lifted oil drained out of the foam structure within short time frame.

Presence of oil, at the volume fraction implemented, affect the stability of the foam columns. The effect depends on the surfactanttype and surfactant concentration where stability decreases at low concentrations of Zonyl FSO and at all concentrations range tested of Hitenol H-10.

Now that Zonyl FSO and Hitenol H-10 were selected as the most effective foaming agents, these two surfactants were investigated in flooding runs. Figure 9 is a plot of oil recovery and total pressure drop across the core sample for water secondary flooding followed by slug

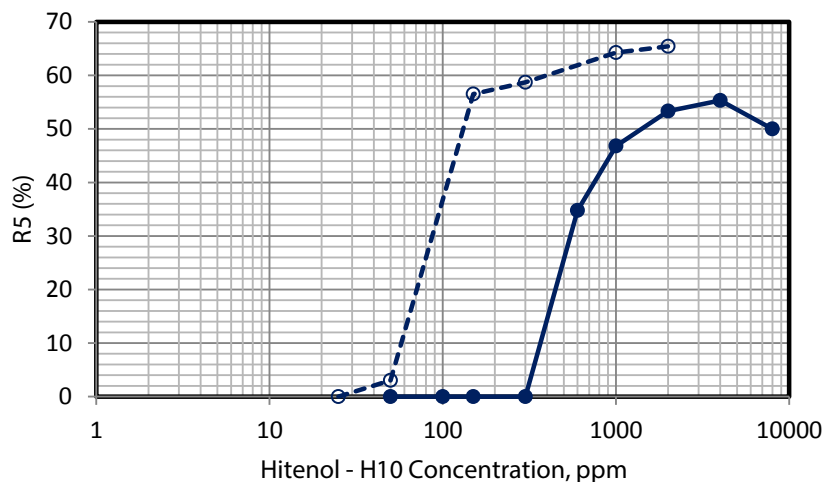

$\longrightarrow$ With oil $-\infty-$ Without Oil

Figure 7: Variation of R5 as a function of surfactant concentration of Hitenol $\mathrm{H}-10$ in presence and absence of oil.

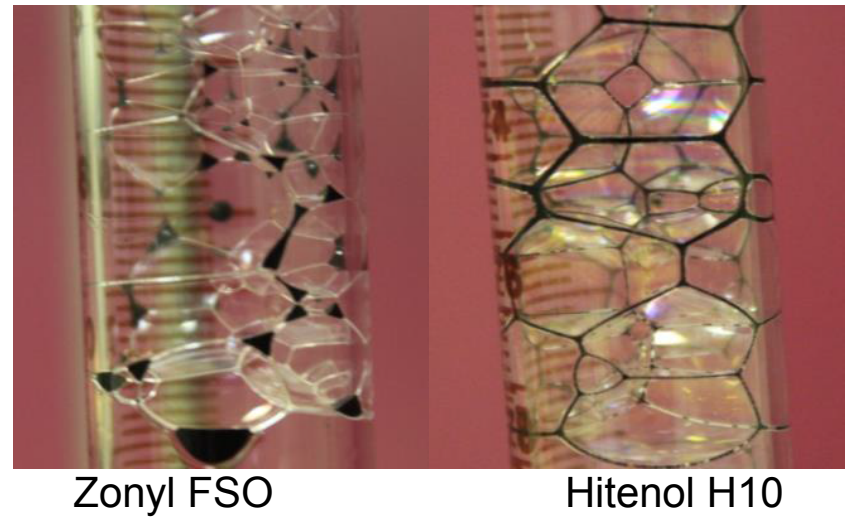

Figure 8: Effect of oil presence on foam structure and stability at $1000 \mathrm{ppm}$ surfactant concentration diluted in $4 \%$ brine. of 1000 ppm Zonyl FSO solution and tertiary gas flooding. Similarly, Figure 10 plots the oil recovery and pressure drop for water secondary flooding followed by a slug of 1000 ppm Hitenol H-10 and tertiary gas flooding. The figures indicate that gas injection in presence of surfactant slug improved oil production by $18.5 \%$ original oil in place (OOIP) for Hitenol H-10 compared to $14.71 \%$ OOIP for Zonyl FSO. That is equivalent to $31.57 \%$ and $28.37 \%$ of residual oil in place for Hitenol $\mathrm{H}-10$ and Zonyl FSO respectively. Comparing the pressure drop across the core for the two runs, Figure 11 explains the relative improved Hitenol $\mathrm{H}-10$ foaming performance as indicated by the higher pressure drop at the experimental conditions applied.

One more experiment was conducted on core sample subjected to secondary water and tertiary gas flooding where residual oil was targeted with 0.2 pore volume of Hitenol $\mathrm{H}-10$ as foaming agent and continuous gas injection. Figure 12 indicates relatively low incremental oil recovery obtained by tertiary gas injection (9\% OOIP) and the role of foaming agent in reducing the gas mobility and improving the displacement efficiency, producing an additional oil recovery of $14 \%$ OOIP. The total incremental oil recovery from this mode of injection was $23 \%$ OOIP, equivalent to $44 \%$ ROIP. Comparing this run with

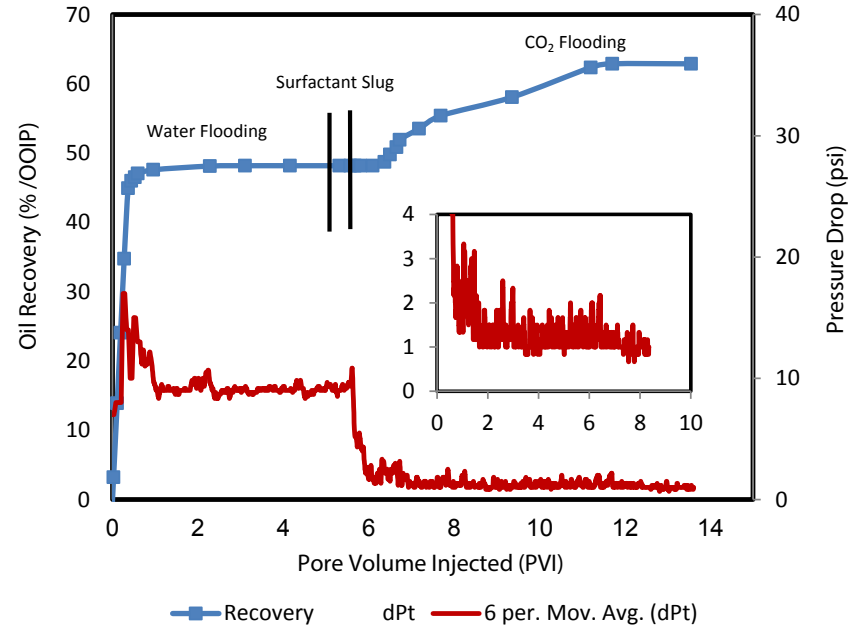

Figure 9: Oil recovery and pressure drop at water flooding, 0.2 PV Zonyl FSO and $\mathrm{CO}_{2}$ injection in presence of light crude oil.

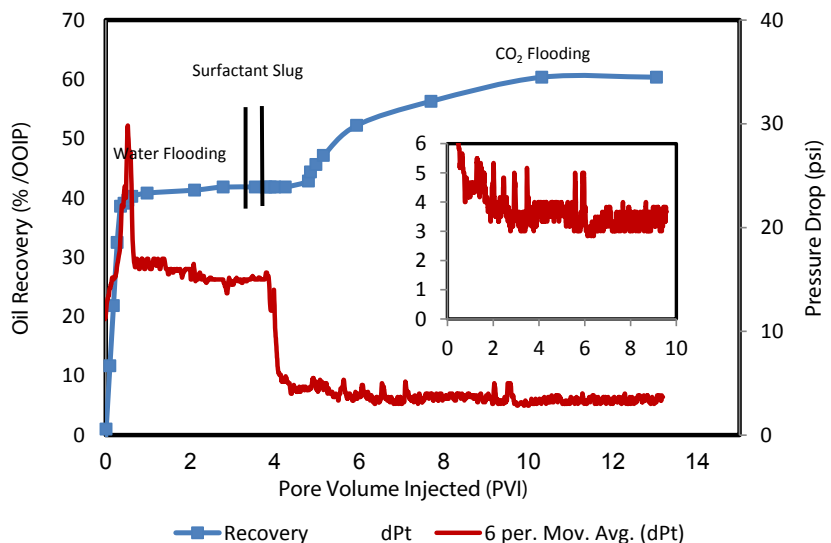

Figure 10: Oil recovery and pressure drop at water flooding, 0.2 PV Hiteno $\mathrm{H}-10$ and $\mathrm{CO}_{2}$ injection in presence of light crude oil. 
Citation: Belhaij A, Mahdy OA (2015) Foamability and Foam Stability of Several Surfactants Solutions: The Role of Screening and Flooding. J Pet Environ Biotechnol 6: 227. doi:10.4172/2157-7463.1000227

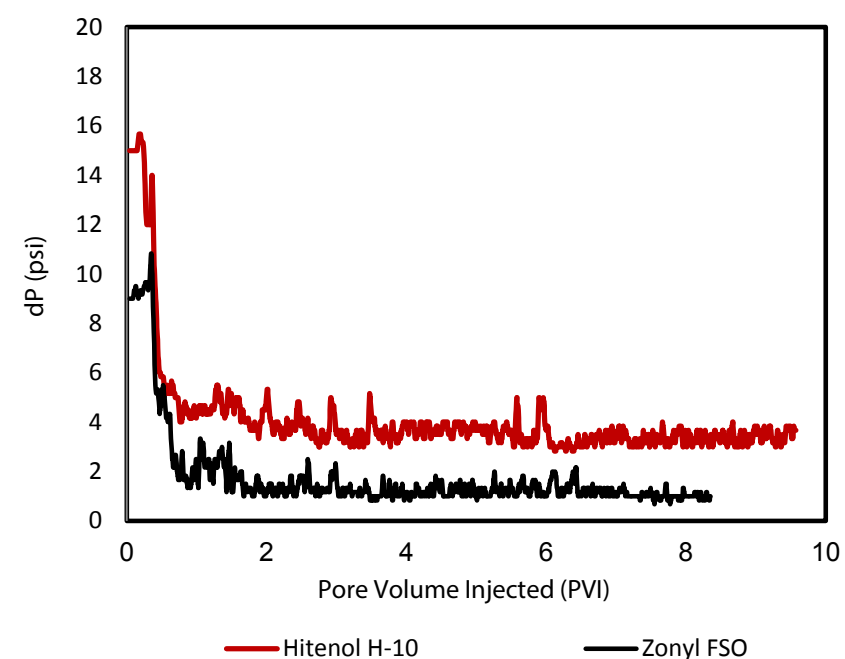

Figure 11: Pressure drop across the core during foam injection for Hitenol $\mathrm{H}-10$ and Zonyl FSO.

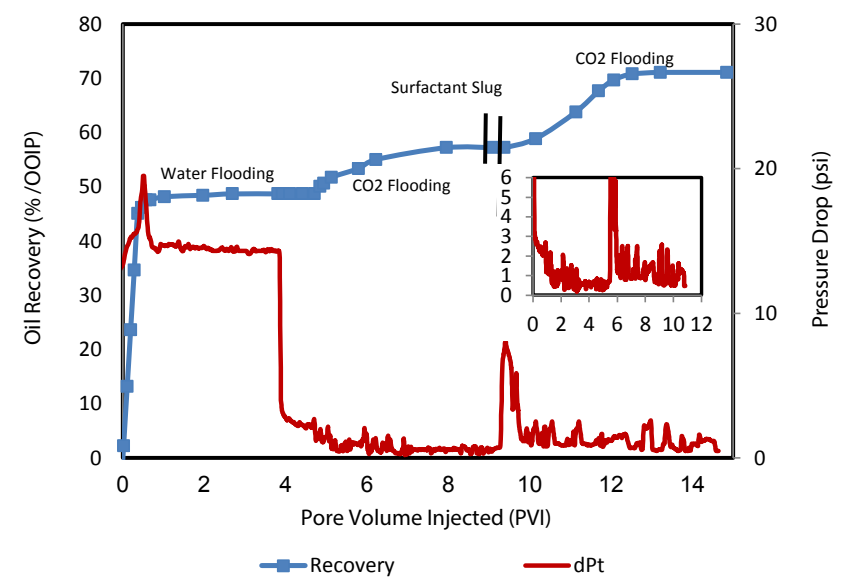

Figure 12: Oil recovery and pressure drop at water flooding, $\mathrm{CO}_{2}$ injection, 0.2 $\mathrm{PV}$ Hitenol $\mathrm{H}-10$ and $\mathrm{CO}_{2}$ injection in presence of light crude oil.

the previous one for Hitenol $\mathrm{H}-10$ discussed earlier, we notice a good performance proving the potential of foam flooding in tertiary gas flooded reservoirs.

\section{Conclusions}

Foamability and foam stability of several anionic and nonionic surfactants were investigated. Foam characteristics were studied at different surfactants concentrations in absence and presence of crude oil. Based on the screening process, the most efficient foaming agent (surfactant) was tested in flooding experiments to test their performance in porous medium. The following are the main outcomes of the work:

1. All surfactants except Triton X-405 showed good formability but Zonyl FSO and Hitenol H-10 surfactants provided the highest foam longevity in absence of oil.

2. Foamability and foam stability enhances as surfactants concentrations increases.
3. High concentration solutions were characterized by fine and fairly uniform distributed bubble size whereas lower concentrations clearly reveals a different foam texture with larger bubble sizes and broad distribution.

4. In presence of oil, lower concentration Zonyl FSO solutions provided lower foam stability. Foam stability was not affected at concentrations above $100 \mathrm{ppm}$. On the other hand, lower stability was noticed at all concentration range tested of Hitenol H-10.

5. Hitenol H-10 surfactant was able to carry good portion of the oil but that oil drained out of the foam structure. To the contrary, oil phase was distributed inside the foam structure of Zonyl FSO accumulating at the plateau borders indicating the strength of foam films.

6. Foam generation in porous medium provided good tertiary recovery controlling gas mobility and enhancing the displacement efficiency. More recovery and higher pressure drop were obtained with Hitenol H-10 indicating its efficiency.

7. Hetinol H-10 Foam injection on tertiary gas flooded reservoirs improves residual oil recovery indicating the potential of the process even at late stages of gas injection.

\section{References}

1. Bond DC, Holbrook OC (1958) Gas drive oil recovery process. U.S. Patent No. $2,866,507$.

2. Fried AN (1961) The Foam Drive Processes for Increasing the Recovery of Oil. No. Report of Investigations 5866, USBM.

3. Bernard GC, Holm LW, Harvey CP (1980) Use of Surfactant to Reduce $\mathrm{CO}_{2}$ Mobility in Oil Displacement. SPE Journal: 281-292.

4. Seright RS, Liang J (1995) A Comparison of Different Types of Blocking Agents, paper SPE 30120, presented at the European Formation Damage Conference held in The Hague, The Netherlands

5. Nguyen QP, Alexandrov AV, Zitha PL, Currie PK (2000) Experimental and Modeling Studies on Foam in Porous Media: A Review. Paper 58799, presented at the SPE International Symposium on Formation Damage Control. Lafayette, LA, pp. 23-24.

6. Farajzadeh R, Wassing BM, Boerrigter PM (2012) Foam assisted gas-oil gravity drainage in naturally fractured reservoirs. Journal of Petroleum Science and Engineering 94-95: 112-122.

7. Schramm LL, Wassmuth F (1994) Foams: Basic Principles. In: Schram -m LL (ed.) Foams: Fundamentals and Applications in the Petroleum Industry. American Chemical Society, Washington, DC, USA.

8. Malysa K, Lunkenheimer K (2008) Foams under dynamic conditions. Current Opinion in Colloid \& Interface Science 13: 150-162.

9. Chiang JC, Sanyal SK, Castanier LM, Brigham WE, Sufi A (1980) Foam as a Mobility Control Agent in steam Injection Processes. Paper SPE 8912 presented at the 50th Annual California Regional Meeting, Los Angeles, USA.

10. Marsden SS, Khan SA (1966) The Flow of Foam through Porous Media and Apparent Viscosity measurements. Soc Pet Eng J 6: 17-25

11. Israelachvili JN (1966) Intermolecular \& Surface Forces (2ndedn) Academic Press, San Diego, 1991. S. S. Marsden, Soc. Pet. Eng. J.

12. Aveyard R, Binks BP, Fletcher PDI, Peck TG, Garrett PR (1993) Entry and spreading of alkane drops at the air-surfactant solution interface in relation to foam and soap film stability. J Chem Soc Faraday Trans 89: 4313-4321.

13. Garrett PR (1993) The mode of action of antifoams, In: Garrett PR (ed.) Deform -ing: theory and industrial applications. Marcel Dekker, New York, USA.

14. Bergeron V, Fagan ME, Radke CJ (1993) Generalized Entering Coefficients-A Criterion for Foam Stability Against Oil in Porous Media. Langmuir 9: 1704-1713.

15. Denkov ND (2004) Mechanisms of foam destruction by oil-based antifoams Langmuir 20: 9463-9505. 
Citation: Belhaij A, Mahdy OA (2015) Foamability and Foam Stability of Several Surfactants Solutions: The Role of Screening and Flooding. J Pet Environ Biotechnol 6: 227. doi:10.4172/2157-7463.1000227

Page 6 of 6

16. Wasan D, Nikolov A, Huang D, Edwards D (1988) Foam stability: effects of oil and film stratiication. In: Smith DH (ed.) Surfactant - based mobility control progress in miscible flood enhanced oil recovery. American Chemical Society, Washington, DC, USA

17. Vikingstad AK, Skauge A, Høiland H, Aarra M (2005) Foam-oil interactions analyzed by static foam tests. Colloids and Surfaces A: Physicochem Eng Aspects 260: 189-198.

18. Nikolov AD, Wasan DT, Huang DW, Edwards DA (1986) The effect of oil on foam stability: mechanisms and implications for oil displacement by foam in porous media. Paper SPE15443-MS presented at The Annual Technical
Conference and Exhibition, 5-8 October 1986, New Orleans, Louisiana.

19. Lau HC, O'Brien SM (1988) Effects of spreading and non-spreading oils on foam propagation through porous media. SPE Reservoir Engineering: 893-896.

20. Simjoo M, Rezaei T, Andrianov A, Zitha PLJ (2013) Foam stability in the presence of oil: effect of surfactant concentration and oil type. Colloids and Surfaces A: Physicochem. Eng Aspects 438: 148-158.

21. Lunkenheimera K, Malysa K (2003) Simple and Generally Applicable Method of Determination and Evaluation of Foam Properties. Journal of Surfactants and Detergents 6: 69-74. 